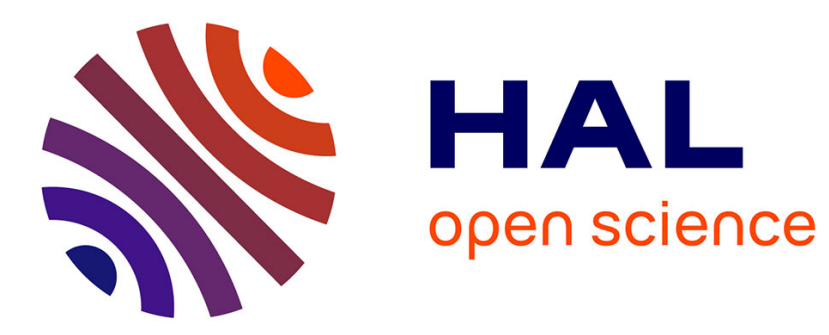

\title{
Des besoins particuliers des élèves aux besoins de formation des professionnels : l'exemple de la déficience visuelle
}

\author{
Nathalie Lewi-Dumont
}

\section{- To cite this version:}

Nathalie Lewi-Dumont. Des besoins particuliers des élèves aux besoins de formation des professionnels : l'exemple de la déficience visuelle. La nouvelle revue de l'adaptation et de la scolarisation, 2015, 2-3 (70-71), pp.149-164. hal-01663070

\section{HAL Id: hal-01663070 \\ https: / hal-inshea.archives-ouvertes.fr/hal-01663070}

Submitted on 13 Dec 2017

HAL is a multi-disciplinary open access archive for the deposit and dissemination of scientific research documents, whether they are published or not. The documents may come from teaching and research institutions in France or abroad, or from public or private research centers.
L'archive ouverte pluridisciplinaire $\mathbf{H A L}$, est destinée au dépôt et à la diffusion de documents scientifiques de niveau recherche, publiés ou non, émanant des établissements d'enseignement et de recherche français ou étrangers, des laboratoires publics ou privés. 


\title{
Des besoins particuliers des élèves aux besoins de formation des professionnels: l'exemple de la déficience visuelle
}

\author{
Nathalie LEWI-DUMONT \\ INS HEA - Grhapes \\ Suresnes
}

Résumé: La déficience visuelle (malvoyance et cécité), rare chez les jeunes, engendre généralement deux types de difficultés pour la scolarisation: dans le domaine de l'espace, du point de vue de l'autonomie et des disciplines le mettant en jeu et dans l'accès aux documents écrits et imagés. Outre les professionnels spécialisés qui s'occupent d'autres types d'élèves handicapés, des intervenants spécifiques pour la locomotion, les activités de la vie journalière et les transcriptions travaillent en interaction et en complémentarité avec l'ensemble de l'équipe afin de répondre aux besoins des élèves. À partir de diverses sources, dont les points de vue de plusieurs acteurs, on montre l'importance de la prise en compte des besoins particuliers par les professionnels. Les vingt jeunes déficients visuels interviewés ont insisté sur cette dimension partenariale: I'enseignant spécialisé a un rôle-clé dans leurs parcours pour favoriser leur inclusion en classe ordinaire et dans leur réussite, sur le plan scolaire et social.

Mots-clés: Adaptation - Autonomie - Braille - Cécité - Déficience visuelle - Espace - Formation - Inclusion.

From pupils' special educational needs to professionals' training needs: the visual impairment example

Summary: Visual impairment (low vision and blindness), a rare handicap among youth, generally generates two types of difficulty for schooling: regarding space (autonomy and school subjects where space is particularly important) and access to print and images. In addition to the professionals who take care of other types of handicapped students, specialized professionals (training in orientation and mobility, in daily living skills and transcribers) work in interaction and complementarity with the whole team to meet the students' needs. From various sources, including several actors' points of views, we show the importance of taking into account the special needs by professionals. In their interviews, 20 young visually impaired emphasized this partnership dimension: the specialist teacher for visually impaired has a key role in their schooling paths to promote their inclusion in regular classes and in their success, both academically and socially.

Keywords: Adaptation - Autonomy - Blindness - Braille - Inclusion - Space - Training - Visual impairment. 
ES enseignants sont censés pouvoir enseigner à l'ensemble des élèves, dans leur diversité, qu'elle soit liée à leur culture ou aux conséquences de troubles physiques, sensoriels ou mentaux (Assude et Perez, 2014; Dorison et LewiDumont, 20111). Depuis 2005, de plus en plus d'élèves handicapés sont inclus, y compris dans l'enseignement secondaire ${ }^{2}$. Parmi ces élèves, les enseignants ont moins l'occasion de recevoir dans leurs classes ceux qui sont atteints de déficits sensoriels, du fait de leur rareté et peu de formations existent. Dans cette population, les jeunes déficients visuels (malvoyants et aveugles) sont les moins nombreux (Inserm, 2002) et connaissent une situation particulière, voire paradoxale. Contrairement aux autres élèves handicapés, ils ont depuis longtemps été intégrés, à la fin du secondaire et à l'université, et pour les aveugles souvent la scolarité en milieu ordinaire venait après l'établissement spécialisé d'où ils sortaient en maîtrisant les techniques de compensation. II s'agissait en général d'élèves particulièrement brillants, bien soutenus par leurs familles, tel, au début du XXe siècle, Pierre Villey, professeur à I'université de Caen, auteur d'une thèse toujours actuellement consultée ${ }^{3}$. Or, la comparaison des statistiques annuelles du ministère de l'Éducation nationale montre que le nombre d'élèves déficients visuels inclus a tendance à baisser, contrairement à ce qui se produit pour d'autres élèves handicapés ${ }^{4}$. Le manque de personnels spécialisés dédiés à la déficience visuelle, notamment les enseignants, est souvent un frein à l'inclusion et peut amener un retour des élèves en dispositif non inclusif, et, en période de contraintes budgétaires, des considérations uniquement sur le nombre d'élèves sont susceptibles de faire oublier qu'ils ne peuvent réussir sans maîtriser des techniques qui s'enseignent ${ }^{5}$.

Après avoir rappelé les contraintes qui se posent pour étudier cette population, je présenterai la spécificité de ses besoins. Je relierai ces besoins aux compétences spécifiques que ces jeunes doivent acquérir pour devenir autonomes dans leurs apprentissages et dans leur vie quotidienne et m'interrogerai sur leurs rapports avec celles que les professionnels doivent posséder pour les y aider dans un contexte inclusif, afin de montrer la complémentarité des approches, et en présentant le point de vue de jeunes déficients visuels sur ce partenariat.

1. Cf. Référentiel des compétences professionnelles des métiers du professorat et de l'éducation, arrêté

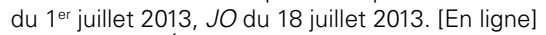

2. Ministère de l'Éducation nationale, de l'Enseignement supérieur et de la Recherche. (2014). Repères et références statistiques sur les enseignements, la formation et la recherche. [En ligne]

3. Les sources et l'évolution des Essais de Montaigne. Paris: Hachette, 1908.

4. Comparaisons entre les données de 2014 et des années antérieures de Repères et références statistiques sur les enseignements, la formation et la recherche.

5. On peut prendre pour exemples deux départements pionniers dans l'intégration individuelle des élèves déficients visuels (malvoyants et aveugles). Dans les années quatre-vingt, plusieurs enseignants spécialisés itinérants intervenaient dans le Calvados et le Val-de-Marne (Cf. L'enfant déficient visuel: conditions de scolarisation. Le courrier de Suresnes, 32, 1981); il n'y en a plus qu'un en 2015 pour soutenir les élèves inclus dans le primaire et un dans le secondaire dans Calvados, et un dans le Val-de-Marne pour l'ensemble des élèves en inclusion individuelle (source: auteur). 


\section{CONSIDÉRATIONS MÉTHODOLOGIQUES: LA DIVERSITÉ DES DÉFICIENCES VISUELLES}

\section{Différents degrés de déficience visuelle}

Il existe différents degrés de déficience visuelle et différentes façons de la déterminer. La définition la plus couramment utilisée est celle de l'Organisation mondiale de la santé (2012) : la cécité correspond à une acuité visuelle corrigée inférieure à 1/20 ou à un champ visuel inférieur à 10 degrés, la malvoyance à une acuité visuelle comprise entre 1/20 et $3 / 10$ ou à un champ visuel d'au moins 20 degrés, avec deux catégories de malvoyance et trois au sein de la cécité (de quelques perceptions lumineuses à une perception nulle). On peut être catégorisé comme aveugle et pouvoir utiliser sa vision dans certaines circonstances. Ces données chiffrées, extrêmement utiles, ne suffisent pas pour comprendre le fonctionnement d'une personne: elle peut avoir une bonne vision périphérique et donc se montrer à l'aise dans ses déplacements, en revanche être très gênée si elle doit écrire ou lire un texte. Par ailleurs, la vision des personnes malvoyantes fluctue souvent en fonction de l'intensité lumineuse, voire de leur fatigue.

À ces comportements liés à l'atteinte visuelle, s'ajoutent d'autres paramètres, notamment le moment de la survenue de cette atteinte. Les recherches en psychologie cognitive (Hatwell, 2003) montrent que les conséquences sur le développement sont plus importantes si la déficience survient à la naissance ou avant l'âge d'un an. Un autre facteur important est l'étiologie de la déficience, qui peut être d'origine génétique ou accidentelle, ou survenir à la suite d'une maladie. Certaines pathologies visuelles peuvent s'accompagner d'autres troubles: actuellement, du fait des progrès médicaux, des enfants nés très prématurément sont atteints de troubles visuels parfois importants mais peuvent aussi souffrir d'autres troubles (moteurs, mentaux) qu'on ne décèle pas toujours dès la naissance.

Le caractère évolutif ou non de la pathologie visuelle est un autre élément à prendre en compte: certaines affections restent stables, d'autres au contraire impliquent une dégradation progressive de la vue, ce qui provoque, notamment à l'adolescence, une grande inquiétude et une réorganisation des projets d'études ou de vie (Blatgé, 2012; Sémelin, 2007).

Ces éléments ont des retentissements différents selon le milieu de vie de l'enfant, de ce que lui-même et ses parents ont pu mettre en place, des accompagnements reçus et les différences entre individus rendent souvent difficiles les généralisations.

\section{Quelques problèmes posés par l'étude de jeunes déficients visuels}

Les études sur les jeunes déficients visuels posent des problèmes méthodologiques et éthiques: puisque la prévalence de la déficience visuelle est la plus faible parmi les handicaps chez l'enfant et l'adolescent, les pathologies survenant plus souvent au cours de la vie ${ }^{6}$, les besoins des élèves malvoyants et aveugles sont difficiles à

6. La maladie la plus fréquente est actuellement la Dégénérescence maculaire liée à l'âge (DMLA). Parmi les maladies pouvant provoquer la malvoyance ou la cécité, le glaucome et la cataracte peuvent être congénitaux mais aussi survenir tardivement, chez des personnes âgées notamment pour la cataracte. La rétinite pigmentaire est souvent diagnostiquée au moment de l'adolescence ou à l'âge adulte. 
étudier scientifiquement. Peu d'enfants naissent aveugles complets sans handicap associé dans les pays développés (cf. Inserm, 2002) et les malvoyants sont une population hétérogène qui, de plus, ne souhaite pas se singulariser. II est dès lors assez difficile d'étudier ces jeunes, faute de trouver un échantillon homogène; en outre, du fait de la rareté du handicap, les enfants et les parents sont souvent sollicités et parfois refusent de participer à des études et même de répondre à des questionnaires. Même dans une revue américaine comme Journal of Vision Impairment and Blindness, les recherches publiées portent sur des sujets hétérogènes (date de survenue de la déficience, degré de déficience, âge, handicap associé ou non) ou présentent des études de cas. Il est nécessaire d'avoir recours, en complément des études expérimentales, à des études écologiques ou qualitatives, qui sont des moyens de connaître les élèves et les professionnels, souvent par l'analyse de corpus longs et/ou longitudinaux.

\section{Sources utilisées}

Outre les références aux écrits scientifiques ou professionnels concernant le développement des jeunes déficients visuels, leur accompagnement et les techniques de compensation les concernant, je m'appuie ici sur des interviews et des échanges écrits. Pour élaborer certaines parties de l'ouvrage collectif (2015, à paraître) Enseigner à des élèves malvoyants, enseigner à des élèves aveugles, j'ai utilisé des interviews et des témoignages de professionnels, de parents et de jeunes déficients visuels dont certains figurent ici ou dont j'extrais des parties inédites: dans le cadre de mon activité de formatrice ou de chercheuse à I'INS HEA-Grhapes, j'effectue de fréquents déplacements dans différents établissements ou dispositifs, j'ai de nombreux échanges oraux et écrits (par courriel) avec des professionnels, notamment des enseignants, des familles, des élèves et des adultes déficients visuels; j'ai utilisé également des verbatim récents tirés du forum de discussion Enfant aveugle et les corpus recueillis et non exploités issus d'interviews de vingt jeunes déficients visuels interrogés sur leur parcours dans le secondaire et sur les aides et les aménagements obtenus (Lewi-Dumont, 2009b) et les études de cas que j'ai conduites pour la recherche de Rick et Ebersold (2011). Dans le présent article, je m'appuie également sur les commentaires d'élèves, de professeurs du second degré interrogés dans le cadre d'une autre recherche (Lewi-Dumont et al., 2015; Puustinen et al., 2015).

\section{QUELS BESOINS SPÉCIFIQUES POUR LES ÉLÈVES DÉFICIENTS VISUELS?}

Soulignons tout d'abord que la cécité et la malvoyance ne sont pas toujours à présenter en termes de besoins, voire de manques, mais que les enfants peuvent développer des compétences compensatoires. Lorsqu'ils ont surmonté certaines difficultés de représentation, le langage oral est souvent un point fort sur lequel s'appuyer, qui leur permet d'interagir avec les adultes et avec leurs pairs, puis d'accéder à l'abstraction et aux connaissances (Hatwell, 2003; Lewi-Dumont, 2011b).

Il est communément admis (Hatwell, 2003), avec les réserves évoquées ci-dessus quant aux façons personnelles dont les sujets surmontent les difficultés, que 
la déficience visuelle a des retentissements importants dans deux domaines spécifiques qui peuvent engendrer des handicaps au niveau des apprentissages et de la scolarisation.

\section{La construction de l'espace}

Statistiquement, plus la déficience est importante et précoce, plus la construction de l'espace se fait difficilement (Hatwell, 2003). L'enfant qui n'a jamais vu ou qui a vu très peu depuis sa naissance doit se construire une représentation de l'espace à partir du toucher et de l'audition (Gentaz, 2009), il doit reconstruire mentalement I'espace, ce qui représente un coût cognitif très important. On a tendance à sous-estimer le problème chez les malvoyants, qui pourtant rencontrent bien des difficultés, majorées précisément par le fait que leur vision fluctue en fonction des circonstances (luminosité, état général). Chez les élèves scolarisés, quel que soit leur niveau, les conséquences des difficultés spatiales se manifestent principalement dans deux domaines, I'un touchant leur autonomie et leur vie sociale d'élèves, l'autre l'accessibilité à certaines disciplines.

\section{La vie quotidienne d'élève}

Dans la classe et surtout en dehors, ce qui relève des déplacements, du repérage dans un lieu peut être difficile. Cette difficulté, si on n'y prend garde, peut conduire à une grande solitude pour l'élève inclus, à différents niveaux scolaires.

Une maman parle de son fils en grande section de maternelle:

" Il passe [les récréations] la plupart de son temps isolé, ne pouvant pas participer aux jeux mouvementés de ses petits copains, le bruit le dérange.

Cette situation est de plus en plus difficile pour lui, je le sens en souffrance.

II n'a même plus envie de sortir l'heure venue. "

Chez les plus âgés, les déplacements autonomes dans des lieux connus (classe, établissement scolaire) deviennent plus faciles avec l'apprentissage et le temps mais les trajets entre le domicile et l'école, même devenus familiers, nécessitent de la concentration: un élève très malvoyant ou aveugle, sauf s'il va au collège au bras d'un camarade, ne peut pas profiter pleinement d'une conversation amicale en marchant. Certains jeunes malvoyants et aveugles éprouvent le besoin d'un retour dans un dispositif moins inclusif, voire abandonnent leurs études lorsque les efforts à fournir pour leurs déplacements sont trop importants (cf. Lewi-Dumont, 1999, 2009b; Rick et Ebersold, 2011). En tout état de cause, l'autonomie dans la vie quotidienne et dans les déplacements est un des défis majeurs de leur éducation.

\section{Les disciplines mettant en jeu l'espace et sa représentation}

De nombreuses disciplines scolaires impliquent, dès les débuts de la scolarisation, la maîtrise de l'espace: I'éducation physique mais aussi la géographie, la géométrie. Les élèves ont parfois une représentation approximative des objets étudiés. Lors d'une enquête sur la demande d'aide des élèves et la façon dont les professeurs de mathématiques les aident (Lewi-Dumont et al. 2015), plusieurs enseignants, après avoir rempli le questionnaire, nous ont contactées pour partager leurs interrogations, par exemple cet enseignant de collège: 
"X perd progressivement la vue. C'est un élève assez doué en mathématiques. Cependant, il est de plus en plus gêné par la géométrie, utiliser un rapporteur, décomposer une figure. [...] Ce qui est très difficile pour moi, c'est trouver des moyens non visuels pour lui apporter des situations problèmes sensibles. Heureusement, il a des facilités d'abstraction mais je crains qu'il ne s'épuise et soit en difficulté à cause de son handicap assez rapidement. "

Dans les 41 réponses à cette enquête concernant des professeurs de mathématiques du second degré, ceux qui ont ajouté le plus de commentaires libres à leurs réponses sont les professeurs de lycée ayant un élève travaillant en braille, comme si la concentration de difficultés offrait une résistance à l'enseignement de leur discipline et, de ce fait, les intéressait.

\section{L'accès à l'écrit et à l'image}

Le deuxième type de conséquences liées aux déficiences visuelles est l'accès à l'écrit et à l'image. Les obstacles rencontrés sont de deux ordres.

La proportion de livres et de manuels adaptés est faible malgré les progrès des logiciels de transcription et les avancées législatives en la matière ${ }^{7}$, surtout dans le domaine scolaire: les manuels contiennent une très riche iconographie qui ne peut être adaptée mécaniquement. En outre, l'image est travaillée à part entière en tant qu'objet d'étude dans les programmes. Trouver des documents est une difficulté importante pour les enseignants, les parents, les élèves et les étudiants. Les modalités de lecture, grâce aux techniques informatiques, sont variées et peuvent dépendre, pour un élève, du type d'écrit à lire et de ses propres préférences. La technologie permet à présent de transcrire ou d'adapter des textes seuls et, en théorie, les élèves devraient pouvoir en disposer vite: on se rend compte par les échanges de groupes de discussion de parents, par les écrits professionnels des enseignants ${ }^{8}$, que ce n'est pas le cas. L'élève devrait avoir le choix entre une version sur papier, une lecture auditive par le biais d'un lecteur humain, d'un enregistrement voire d'une synthèse vocale ou encore une lecture sur écran avec un logiciel d'agrandissement ou sur une plage tactile braille. En réalité, les élèves lisent en fonction de ce qui existe: les jeunes interviewés rappellent que, même s'ils préfèrent lire sur papier, ils lisent ce qu'ils trouvent.

En outre, la lecture est plus lente, notamment en braille mais même pour les malvoyants, en moyenne la vitesse est trois fois plus lente qu'en noir ${ }^{9}$, puisque le lecteur n'a pas de vision synoptique, ne peut se faire une représentation mentale du texte, procéder par feuilletage, faire une lecture sélective (Lewi-Dumont, 1997). L'image tactile et le dessin en relief font partie maintenant des moyens d'accessibilisatio très utilisés. II peut s'agir d'illustrations par le biais de différentes techniques, y compris

7. Cf. en France la loi $n^{\circ}$ 2009-669 du 12 juin 2009 favorisant la diffusion et la protection de la création sur internet, dite loi Hadopi ou au niveau international le traité de Marrakech (Organisation mondiale de la propriété intellectuelle) " visant à faciliter l'accès des aveugles, des déficients visuels et des personnes ayant d'autres difficultés de lecture des textes imprimés aux œuvres publiées " (27 juin 2013).

8. Dans le groupe de discussion Enfant aveugle, le thème de la difficulté à obtenir des documents adaptés est un des plus fréquents (de la maternelle au lycée), les parents prenant le relais pour que leur enfant ne soit pas pénalisé.

9. Expression utilisée par les aveugles pour désigner l'écriture ordinaire des voyants. 
artisanales (par collage de matériaux) : dans les albums tactiles, le temps d'exploration est très lent et la représentation en relief, même simple (un enfant, une voiture...) n'a pas le même rôle que dans un album illustré en noir, elle n'aide pas le jeune enfant non voyant à décoder le texte mais au contraire est elle-même objet de décodage (Lewi-Dumont, 1997). En histoire, géographie, sciences, les dessins en relief, quels que soient les procédés de réalisation, nécessitent aussi une technique de lecture particulière (Bris, 2015). L'élève découvrant une carte de géographie ou un schéma en sciences a souvent besoin de descriptions complémentaires ou d'un guidage verbal pour étayer cette découverte, qu'il travaille en modalité visuelle ou tactile.

En conséquence, aujourd'hui encore, la réussite du parcours scolaire et universitaire d'un jeune déficient visuel dépend de l'obtention des documents et de la qualité de leur adaptation (Rick et Ebersold, 2011).

La maîtrise de l'espace et l'accès à l'écrit ont pour conséquence une lenteur qui n'est pas dans l'idéation, mais dans la réalisation des tâches (l'exploration tactile est plus lente et, fragmentée, doit ensuite être synthétisée) et qui est accompagnée de manifestations de fatigue liée à la concentration (non à un problème somatique). Ainsi, Noël évoque les raisons de sa demande d'arrêt de la scolarisation en milieu ordinaire ${ }^{10}$ :

" Je faisais que ça [des efforts], et j'en pouvais plus à la fin! Ah! Non, c'était horrible! On est mort de la journée, et puis il y a le trajet et quand on rentre, il faut travailler encore plus que les autres [...] I/ faut canaliser toute son énergie à écouter tous les cours ! [...] à la fin oui, j'étais découragé, j'en pouvais plus. " (Lewi-Dumont, 1999).

\section{DES COMPÉTENCES PROFESSIONNELLES POUR RÉPONDRE AUX BESOINS SPÉCIFIQUES}

\section{Scolarisation de ces élèves et spécificités de leur accompagnement}

Comme tous les élèves en France, les jeunes déficients visuels et leurs parents peuvent choisir entre une inclusion individuelle, une scolarisation en dispositif collectif ou en unité d'enseignement. Le choix n'est pas lié à la gravité de l'atteinte visuelle, des jeunes aveugles complets pouvant avoir une scolarité totalement inclusive, mais aux possibilités d'accompagnement de chaque élève, aux aides techniques dont il a besoin, au temps de trajet s'il n'est pas dans son établissement de référence, etc. On constate néanmoins que les possibilités sont inégalement réparties sur le territoire, les parents n'ayant parfois qu'une seule solution ou devant se résoudre à déménager ou à envoyer leur enfant en internat (Lewi-Dumont, 2011a, 2015 ; Onisep, 2014).

10. II faut souligner une évolution positive dans la perception de la scolarisation des élèves déficients visuels en milieu ordinaire. Par exemple, entre la série d'interviews analysées en 1999 et celle de 2009, si les élèves ou anciens élèves mentionnent encore certains comportements de professeurs ne respectant pas la dimension éthique de leur métier (se livrant par exemple à des remarques blessantes envers les élèves), ces constats sont numériquement moins importants. Dans les réponses des 41 professeurs de mathématiques du secondaire (Lewi-Dumont et al., 2015), nous n'avons eu aucune remarque faisant observer qu'un élève se trouverait mieux dans un établissement spécialisé, même quand un constat de manque d'accompagnement était émis. 
En France, le service spécifiquement dédié à la déficience visuelle est le Service d'aide à l'acquisition de l'autonomie et à la scolarisation (S3AS ou SAAAS). Comme on l'a vu ci-dessus, l'autonomie est un objectif majeur de l'éducation des jeunes déficients visuels, d'où la présence de ce mot dans le nom du dispositif. Ce service est dédié aux jeunes de 3 à 20 ans, à la suite du Service d'accompagnement familial et d'éducation précoce (Safep) pour les enfants de la naissance à 3 ans ${ }^{11}$. Le S3AS suit les élèves bénéficiant d'une inclusion individuelle ou collective mais tous les départements n'en disposent pas (Onisep, 2014). Enfin, une originalité que les élèves déficients sensoriels (déficients auditifs et déficients visuels) partagent est qu'ils peuvent être suivis par des enseignants spécialisés de l'Éducation nationale ou par des professeurs de sourds ou d'aveugles du ministère chargé des Affaires sociales: il s'agit d'une formation en cours d'emploi (sans expérience préalable de l'enseignement) de préparation au Caega-DV (Mithout, 2015 ${ }^{12}$ ).

On rencontre dans les dispositifs ou établissements destinés à de jeunes déficients visuels le même type de professionnels que ceux qui interviennent dans ceux qui s'occupent en général de jeunes handicapés, ceux des secteurs médical, paramédical, éducatif et rééducatif. En revanche, ce sont seulement dans les dispositifs spécifiques à la déficience visuelle qu'interviennent certains professionnels, dans les deux domaines spécifiques évoqués ci-dessus, ils travaillent en partenariat étroit avec leurs collègues de l'équipe pluridisciplinaire et avec les enseignants.

\section{Des professionnels spécifiques}

Les instructeurs de locomotion interviennent à domicile ou sur le lieu de scolarisation pour que l'élève apprenne à mieux s'orienter dans des lieux connus, puis de moins en moins connus, en sécurité. Ces professionnels, qui en France, ne sont pas assez nombreux pour couvrir les besoins ${ }^{13}$, s'appuient sur des maquettes, des dessins adaptés ou en relief pour enfin enseigner la circulation indépendante et la technique de la canne pour ceux qui en ont besoin. Leurs objectifs majeurs sont la sécurité et l'autonomie. Toujours pour que les jeunes accèdent à l'autonomie dans les gestes les plus quotidiens, des professionnels ayant suivi une formation complémentaire initient les enfants aux Activités de la vie journalière (AVJ) : manger proprement et seul, s'habiller, mettre la table, plus tard se maquiller, se raser ou préparer les repas. Ces compétences font largement appel à la maîtrise de gestes moteurs, à la mémoire et au repérage spatiaux, tactiles et auditifs. Un jeune enfant qui apprend à mettre le couvert apprend par là même à se repérer, à se latéraliser, à exercer son toucher. Ces deux métiers sont complémentaires (Heyraud, 2013) et, même s'ils n'ont pas, au départ, l'ambition de favoriser la réussite à l'école, ils peuvent y contribuer, avec l'action des parents et des éducateurs. Tout le travail de repérage sur supports adaptés (maquettes, schémas, etc.) prépare aux apprentissages en géographie par exemple ou en est complémentaire.

11. Code de l'action sociale et des familles.

12. Certificat d'aptitude à l'enseignement général des aveugles et des déficients visuels.

13. Certains S3AS n'ont pas d'instructeurs en locomotion (Onisep, 2014). Selon l'Association des instructeurs en locomotion, pour personnes déficientes visuelles (AILDV), il y avait environ 200 instructeurs en France en 2013 (pour l'ensemble de la population, enfants et adultes). 
De nombreux professionnels, concourent à l'autonomie des élèves dans le domaine de l'écrit. Les enseignants ont en charge l'apprentissage de la lecture et de l'écriture, les ophtalmologistes et les orthoptistes permettent, pour les malvoyants, de tirer parti de leur vision résiduelle en corrigeant ce qui peut l'être et en leur apprenant à l'utiliser le mieux possible. Même si la déficience visuelle à elle seule n'a pas pour conséquence des troubles du langage, il arrive que les enfants soient suivis en orthophonie (Derrier et Fer, 2012). Parfois des ergothérapeutes interviennent, notamment pour l'apprentissage de techniques de compensation, la dactylographie, l'informatique.

En outre, et ces professionnels travaillent uniquement dans les structures dédiées au handicap visuel, les structures doivent se doter d'un service de transcription. Même s'ils ne s'occupent pas directement des élèves, les transcripteurs-adaptateurs ont un rôle dans leur scolarisation: ils adaptent les textes, les ouvrages, les contrôles à la demande des enseignants, y compris les images, schémas, tableaux.

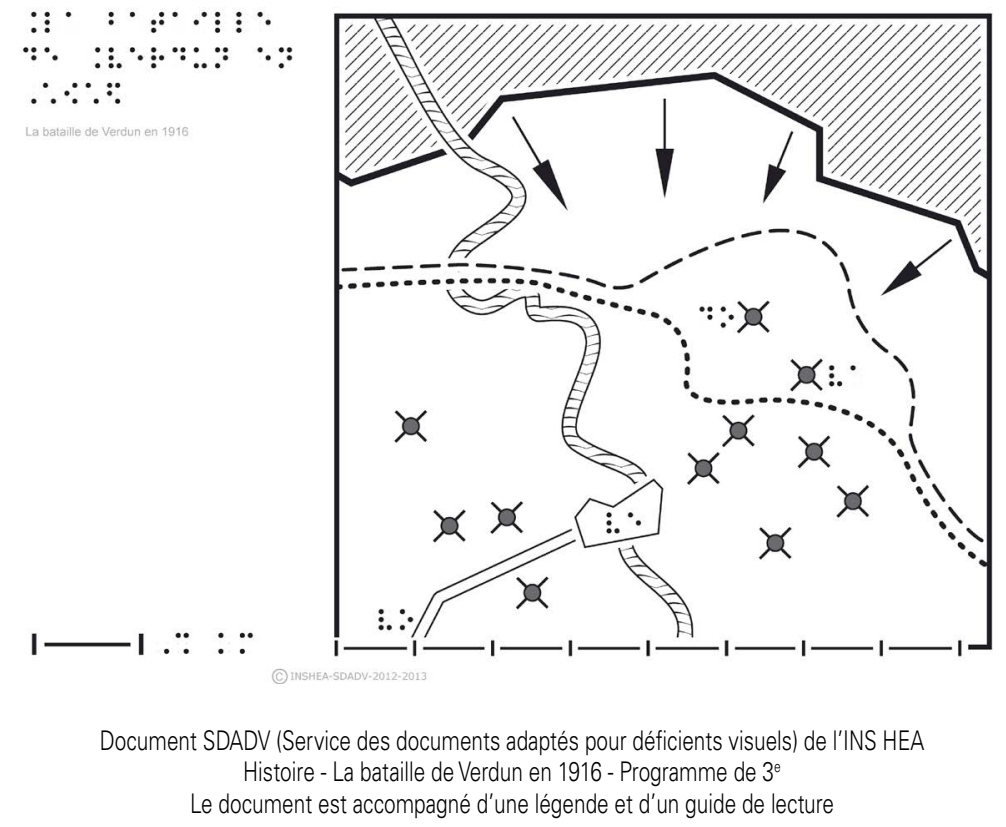

Un des jeunes aveugles interviewés a dû quitter son établissement scolaire de référence à la fin du collège car le service qui le suivait ne disposait que d'un seul transcripteur pour tous les élèves suivis, qui ne parvenait pas à lui fournir tous les documents en braille et en relief sans négliger les autres élèves: son affectation en établissement spécialisé avec internat pour la fin de ses études secondaires avec scolarisation en Unité d'Enseignement ne répond pas à une demande de sa part ou à de trop grandes difficultés à suivre le rythme d'une classe ordinaire, mais seulement à des moyens humains insuffisants (Lewi-Dumont, 2009b). Dans l'ensemble des interviews, les jeunes ayant connu un dispositif inclusif et une structure 
moins inclusive emploient le mot confort pour souligner le fait qu'en établissement spécialisé ils ont le matériel et les adaptations facilement, ce qui n'est pas toujours le cas en inclusion. Les parents évoquent les longues soirées passées à scanner ou à lire des documents pour leurs enfants.

\section{Des actions complémentaires au service des apprentissages}

Ces trois types de professionnels, qu'on ne trouve pas dans d'autres types d'établissements médico-sociaux, travaillent en relation étroite avec les enseignants des classes et les enseignants spécialisés. En fonction des situations locales, il existe des zones de chevauchement des compétences. On peut donner des exemples dans le primaire comme dans le secondaire. Dans un contexte de pénurie de personnels formés, les enseignants mis à disposition d'un S3AS ou dépendant d'une inspection ASH, dits itinérants, qui accompagnent un jeune élève futur lecteur en braille, lui font travailler les compétences préalables spécifiques à la lecture en braille, notamment, les habiletés tactiles, les mouvements bimanuels, la latéralisation (Lewi-Dumont, 1997, 2009a) ; avant d'arriver à la lecture sur papier, le passage par le tridimensionnel est nécessaire et tout ce qui aide l'enfant à reconnaître par le toucher, à s'orienter, à utiliser ses deux mains, y compris dans sa vie quotidienne, est bénéfique. On voit que l'exemple de l'apprentissage de la mise du couvert avec un AVjiste met en jeu toutes ces compétences.

Une psychomotricienne et une instructrice de locomotion montrent dans une vignette clinique un travail effectué avec des élèves aveugles tardifs sur la notion de cercle en lien avec des difficultés en géométrie.

"Les jeunes s'interrogent sur la notion de cercle et de la correspondance avec I'outil qu'est le compas pour le représenter.

Deux professionnels leur proposent donc une mise en situation réelle à l'aide d'une corde, dans un travail à deux. I/ s'agit de réaliser en grandeur nature, le mouvement que réalise le compas, un jeune représentant la pointe, l'autre le stylo. Le déplacement spatial du second représente donc le tracé du cercle, et la rotation du premier lui donne des informations proprioceptives et kinesthésiques d'une grande importance.

La concordance de ces deux expériences, vécues par chacun des jeunes, leur permet de pouvoir ainsi transposer ce concept spatial, avec le vocabulaire qui lui est associé, sur une feuille et sur tout autre support. Ainsi, d'un déplacement ou mouvement ample qui engage l'ensemble du corps, ils peuvent faire le lien avec un circuit plus fin de la main, des doigts ou d'un objet tel que le compas. " (Toubert et Bartolucci, 2015).

Si un enseignant spécialisé n'a pas vocation à passer une grande partie de son temps à transcrire ou adapter des documents, de facto, presque tous y consacrent une partie de leurs activités, notamment pour des documents courts ou envoyés hors des délais demandés par les services de transcription. De plus, et c'est un rôle particulièrement intéressant, l'enseignant spécialisé est souvent une sorte d'interface entre le service de transcription et le professeur demandant une adaptation (Blancher, 2010). L'enseignant spécialisé, même non spécialiste de chaque discipline, connaît les contraintes de l'adaptation technique et les compétences et connaissances 
devant être maîtrisées par l'élève. Faute de cet échange entre le transcripteur et un pédagogue (qui pourrait être le professeur de la classe, mais cet idéal est rarement atteint), les adaptations de documents perdent souvent de leur pertinence.

II ne suffit pas pour l'élève d'avoir un document adapté pour savoir le lire et le comprendre en classe, surtout lorsqu'il est jeune. Les orthoptistes, les orthophonistes quelquefois, les ergothérapeutes pour le braille, les éducateurs parfois en complémentarité avec les enseignants (spécialisés ou non) sont chargés, entre autres, de rendre l'élève autonome dans les tâches de lecture écriture, avec pour certains un versant rééducatif et pour d'autres plus pédagogique, qu'il s'agisse de l'apprentissage des aides optiques ou des aides électroniques, notamment informatiques.

\section{Un exemple: le braille}

Le braille est un code qu'il ne suffit pas de maîtriser pour savoir l'enseigner, même si c'est la première condition. À propos de la décision de son apprentissage, du passage au braille abrégé ${ }^{14}$, I'enseignant spécialisé, au sein d'une équipe, et particulièrement dans un contexte inclusif, est à même de transmettre les contraintes liées à cet apprentissage. Certes un enseignant non spécialisé peut apprendre le braille intégral, mais avec quelques dizaines d'autres élèves, pourra-t-il adapter sa méthode aux contraintes du braille et adapter les exercices et la progression en tenant compte de la présence de cet élève (Lewi-Dumont, 2009a) ? Cela n'est pas impossible mais reste difficile, car on a du mal à imaginer tous les professeurs des écoles transposant les exercices de reconnaissance visuelle en exercice de reconnaissance tactile, c'est-à-dire gardant l'objectif mais l'adaptant, introduisant de nouvelles lettres qui posent très peu de difficultés à l'ensemble de ses élèves, mais qui doivent être enseignées systématiquement en braille, par exemple la lettre è :: qui ressemble si peu à la lettre e :: ? Un enseignant ayant enseigné en milieu ordinaire et ayant une formation à la déficience visuelle peut faire le lien entre les apprentissages et proposer une progression cohérente en inclusion. Il est censé savoir quand supprimer, quand modifier, quand contourner, quel outil ou quel support choisir pour quel exercice. Si on adopte le point de vue des parents, ce genre de décision n'est pas de la responsabilité d'un accompagnant mais bien d'un enseignant. Un accompagnant pourra bien entendu conforter les apprentissages. De la même façon, et toujours en prenant pour exemple un contexte inclusif, lorsque l'élève est en mesure de commencer d'autres apprentissages exigeants, tels que l'informatique adaptée ou le braille abrégé, un enseignant spécialisé peut enseigner les techniques non pas en tant que telles mais en tant que moyens d'apprentissages scolaires, d'autant plus que le numérique fait partie des priorités actuelles ${ }^{15}$. Certes un ergothérapeute peut avoir des compétences en braille, en informatique et en orthographe, mais relier informatique et enseignement de l'orthographe reste du domaine de compétences des enseignants, car il ne suffit pas de savoir pour savoir enseigner.

\footnotetext{
14. Le braille intégral est un transcodage lettre à lettre, l'abrégé, beaucoup plus complexe, est une sorte de sténographie avec de nombreuses règles, qui permet un gain de place pour les documents imprimés, une augmentation de la vitesse d'écriture et d'écriture lorsqu'il est bien maîtrisé. Les compétences en braille abrégé sont actuellement validées pour devenir enseignant spécialisé pour les élèves déficients visuels. 15. Cf. loi du 8 juillet 2013 pour la refondation de l'École.
} 


\section{PROFESSIONNALISME ET PARCOURS DE SCOLARISATION: LE POINT DE VUE DES INTÉRESSÉS}

Tous les professionnels spécifiques n'interviennent pas auprès des élèves, parfois un élève peut avoir besoin d'acquérir de nombreuses techniques de compensation ou a besoin de certaines rééducations, mais les équipes, en liaison avec les parents, doivent hiérarchiser les priorités pour éviter une surcharge d'emploi du temps. La pluridisciplinarité et la coordination des différentes actions spécifiques sont souvent le gage de la réussite globale des projets des jeunes, en termes de résultats scolaires mais aussi d'autonomie sociale.

\section{Importance des compétences professionnelles spécifiques et de leur interaction}

Interrogés sur leurs parcours dans le secondaire (Lewi-Dumont, 2009b), vingt jeunes déficients visuels, avec recul, se sont souvent référés à des difficultés, notamment de communication. Certains pensent que leur orientation a été proposée plutôt en fonction des filières existantes ou de parcours tracés par des prédécesseurs, voire pour assurer un certain confort de fonctionnement des professionnels plutôt qu'en fonction de leurs propres besoins ou objectifs. Quand une carence en personnel, par exemple en locomotion ou en transcription, a existé, ils font remarquer que cela a eu des conséquences soit sur leur orientation, soit sur leur vie sociale.

Ainsi, Patricia regrette de n'avoir pas eu de locomotion au début de sa scolarité secondaire dans un dispositif collectif:

" J'étais tout le temps avec des amis voyants et forcément j'étais tout le temps à leur bras, j'pouvais pas faire autrement, le collège je le connaissais par cœur donc même si je devais circuler seule, sans canne j'y arrivais sans problème, mais c'est vrai que c'est après, quand j'ai grandi, que j'ai dû faire des sorties un peu plus fréquentes avec des copains tout ça que ça devenait plus compliqué pasqu'il fallait toujours que j'aie quelqu'un pour m'accompagner. " Scolarisée ensuite en établissement spécialisé, elle ne s'y sent pas bien et aurait souhaité revenir en milieu ordinaire:

"Sincèrement ils [l'établissement spécialisé] ont mis un frein pour la locomotion, et après ils m'ont dit que je pouvais pas [être intégrée] parce que je pouvais pas aller au collège seule." "

S'ils ont eu ont eu parfois à souffrir de certaines rivalités entre les adultes, ces jeunes pensent que le partage des compétences est possible et bénéfique. Ils analysent avec recul le fait qu'une mauvaise coopération entre spécialistes et enseignants se fait à leur détriment. Simon, élève de $1^{\mathrm{re}}$, dit qu'il préfère s'adresser directement à ses professeurs et ne veut personne pour faire écran. Paméla, étudiante qui eu une scolarité complète en inclusion, répète à plusieurs reprises qu'elle a " eu de la chance " (avec l'ensemble de ses enseignants, ses AVS) et explique que " souvent ses professeurs réfléchissaient directement avec [l'enseignante spécialisée] aux possibles adaptations ».

Lorsque les interactions entre professionnels sont de qualité, les jeunes valorisent l'absence de rupture au moment des transitions (passage au collège, au lycée, etc.). En revanche, quand des conflits de pouvoir (dans plusieurs cas, entre milieu ordinaire et dispositif spécialisé) existent ou encore une absence de choix dans les modalités de scolarisation, plusieurs déplorent d'en avoir souffert. 


\section{Des conditions pour la réussite des parcours}

Dans tous les entretiens, plusieurs points sont systématiquement abordés par les jeunes interviewés.

La question de départ concernait les parcours de scolarisation à partir du collège, mais les jeunes interrogés se sont systématiquement référés à ce qui la précédait et à l'importance de la littéracie, ce qu'ils appellent les bases, inculquées le plus souvent par leur enseignant spécialisé. Ralph notamment a beaucoup insisté sur les apprentissages de l'école primaire et conclut: " si on rate le primaire, c'est un peu foutu ".

Le deuxième point évoqué concerne les aspects matériels, notamment les écrits adaptés, dont l'absence ou l'arrivée trop tardive peuvent avoir de lourdes conséquences à toutes les étapes de la scolarité, voire de la vie en général. Jane explique qu'on ne lui a pas permis d'avoir une imprimante braille durant ses études d'espagnol:

"Moi j'ai exprimé mes besoins et elle [la personne qui déterminait les aides matérielles] m'a dit: vous verrez, vous vous y ferez! ... et j'm'y suis tellement faite que j'ai abandonné l'espagnol... Enfin moi je trouve que faire un commentaire de texte à l'ordinateur, y en a qui y arrivent mais moi je préférais vraiment euh avoir du braille sous les doigts." "

Le dernier point est moins directement lié à la question ici traitée : les jeunes revendiquent la possibilité d'avoir un choix dans la variété des dispositifs et un respect de leurs demandes et de leurs aspirations, ce qui renvoie à l'idée de parcours énoncée par la loi 2005-102. Ils emploient le mot confort pour parler des dispositifs spécialisés et au contraire pour parler de l'intégration, le mot efforts, qui désigne à la fois ceux des élèves et ceux des professionnels, qu'il s'agisse de leurs professeurs ou des professionnels spécialisés. C'est, indirectement, à la concertation et au partage des informations dans le respect de leurs besoins qu'ils font allusion, le mot confiance dans leurs capacités étant également prononcé par plusieurs d'entre eux.

\section{CONCLUSION}

Des exemples de réussite académique et professionnelle d'élèves malvoyants et aveugles peuvent laisser penser que leurs besoins d'accompagnement ne sont pas importants et que les technologies numériques, si indispensables pour eux, peuvent actuellement résoudre tous leurs problèmes d'apprentissage et d'autonomie. Des défaillances dans l'éducation à l'autonomie, des documents écrits et imagés non adaptés ou mal adaptés peuvent avoir des conséquences sur le devenir des élèves déficients visuels. Nous avons montré que des professionnels formés, s'ils sont en nombre suffisant, ont des compétences spécifiques permettant de répondre aux besoins individuels de l'élève, qui ne sont pas que scolaires. Comme le souligne Mithout (2015), il existe un transfert de compétences du milieu spécialisé vers le milieu ordinaire: I'enseignant spécialisé, dans un contexte inclusif ou en établissement spécialisé, doit s'adapter à des besoins très divers et en évolution. Le risque est que, comme au Japon, les enseignants spécialisés se trouvent de moins en moins en situation d'enseigner face aux élèves handicapés, ce qui aboutit au retour des élèves vers des structures spécialisées ou que, dans le contexte français, on admette que l'essentiel des apprentissages scolaires se fasse par le truchement de personnels non enseignants. 
Valentin Haüy, philanthrope voyant qui a ouvert à Paris la première école pour aveugles au monde et a inventé pour eux un système d'écriture en relief, écrivait en 1786: "En toutes choses, il faut rapprocher l'aveugle du voyant. "Quelques dizaines d'années plus tard, un jeune aveugle, Louis Braille, a fait triompher un autre système qui porte son nom, plus difficile à décoder pour un voyant parce que non plus fondé sur des lettres de l'alphabet latin en relief mais sur des points, bien mieux discriminables au doigt. Si on fait un parallèle, la tentation est grande aujourd'hui de faire passer les aveugles au tout numérique (notamment par le biais de l'audio) et de vouloir qu'ils aient tous le même cheminement dans les apprentissages, puisqu'ils sont scolarisés avec tous. Cette facilité est un risque évoqué par tous les protagonistes de la scolarisation des élèves déficients visuels, y compris les associations: ne pas avoir un enseignement de l'écrit dispensé par un personnel qualifié risquerait de les faire revenir avant l'époque de Haüy où ils ne maîtrisaient pas l'écriture.

À un moment où une réforme de la formation spécialisée des enseignants est annoncée, il est nécessaire, à notre sens, d'insister sur les besoins communs à tous les élèves dans leur contexte de scolarisation sans gommer les différences entre eux, ce qui donne tout son sens au syntagme besoins éducatifs particuliers: la maîtrise de certaines techniques ou savoirs spécifiques par les enseignants spécialisés et leur transmission permettront une meilleure maîtrise des compétences scolaires par l'élève. Un raisonnement analogue pourrait être conduit avec d'autres profils d'élèves. Le choix des élèves déficients visuels comme population exemple est lié au fait que leur rareté peut les faire oublier ${ }^{16}$. Ils sont peu présents, contrairement aux élèves sourds, dans les rapports récents qui proposent des mesures intéressantes pour améliorer la qualité de la scolarisation (Blanc, 2011), notamment sur la formation des personnels. C'est surtout grâce à des adaptations réfléchies, à des aides techniques enseignées par des personnels qualifiés parvenant à travailler en complémentarité qu'ils réussissent leur parcours scolaire puis professionnel.

16. À la fin de la rédaction de cet article (juin 2015), des courriels longs et virulents de parents d'enfants malvoyants et aveugles sont échangés sur le forum Enfant aveugle, ne souhaitant manifestement pas un amalgame avec tous les enfants handicapés: " notre problème pour être entendus est que le handicap visuel est largement minoritaire, et qu'on est rapidement assimilés et noyés avec d'autres handicaps sans aucun rapport "; "On a des intérêts communs mais on représente un handicap tellement minoritaire qu'on se fait bouffer." 


\section{Références}

Assude, T. et Perez, J.-M. (2014). Savoirs professionnels et pratiques inclusives. La nouvelle revue de l'adaptation et de la scolarisation, 65, 5-204.

Blanc, P. (Rapp.). (2011). La scolarisation des enfants handicapés. Rapport au Président de la République. Paris: La Documentation Française.

Blancher, M. (2010). Un travail d'enseignant itinérant. La nouvelle revue de l'adaptation et de la scolarisation, 51, 67-78.

Blatgé, M. (2012). Apprendre la déficience visuelle. Grenoble: PUG.

Bris, M. (2015, à paraître). L'utilisation des documents graphiques avec des élèves déficients visuels. In N. Lewi-Dumont (dir.). Enseigner à des élèves malvoyants, enseigner à des élèves aveugles. Lille: Canopé.

Derrier, S. et Fer, A. (2012). L'orthophoniste et l'enfant déficient visuel. L'Ortho Magazine, 102, 13-32.

Dorison, C. et Lewi-Dumont, N. (2011). La formation de tous les enseignants à la diversité. La nouvelle revue de l'adaptation et de la scolarisation, 55, 7-228.

Gentaz, É. (2009). La main, le cerveau et le toucher. Paris: Dunod.

Hatwell, Y. (2003). Psychologie cognitive de la cécité précoce. Paris: Dunod.

Haüy, V. (1786-1985). Essai sur l'éducation des aveugles. Paris: Éditions des archives contemporaines.

Heyraud, J. (2013). L'accompagnement au quotidien des personnes déficientes visuelles. Toulouse: Érès.

Inserm. (2002). Déficits visuels: dépistage et prise en charge chez le jeune enfant. Paris: Inserm.

Lewi-Dumont, N. (1997). L'apprentissage de la lecture chez les enfants aveugles. Difficultés et évolution des compétences. Lille : Septentrion,

Lewi-Dumont, N. (1999). "Moi je n'ai pas envie de prendre ce qui reste". Proclamation de l'identité et de l'altérité chez des adolescents déficients visuels. La nouvelle revue de I'AIS, 4, 66-77.

Lewi-Dumont, N. (2009a). Exercices et jeux de lecture en braille [cédérom, fac-similé des fichiers à embosser, livret pédagogique]. Suresnes: INS HEA.

Lewi-Dumont, N. (2009b). Blind teen-age students' expectations. Proceedings of the 7th European Conference of the International Council for Education of People with Visual Impairment (ICEVI), 5-10 juillet, Dublin.

Lewi-Dumont, N. (2011a). Éducation et enseignement. Repères culturels de la cécité. $\forall$ ir, 38-39. Bruxelles: Ligue Braille, p. 98-105.

Lewi-Dumont, N. (2011b). Langage . Repères culturels de la cécité. Voir, 38-39. Bruxelles: Ligue Braille, p. 174-184.

Lewi-Dumont, N. (2015, à paraître). Enseigner à des élèves malvoyants, enseigner à des élèves aveugle. Lille: Canopé.

Lewi-Dumont, N., Arneton, M. et Puustinen, M. (2015). Les conditions d'enseignement dans le secondaire: analyse de l'adaptation des professeurs de mathématiques aux spécificités des élèves déficients visuels. Colloque Condition(s) enseignante(s), Conditions pour enseigner, Université de Lyon 2, 8-10 janvier. 
Ministère de l'Éducation nationale, de l'Enseignement supérieur et de la Recherche. (2014). Repères et références statistiques sur les enseignements, la formation et la recherche. [En ligne]

Mithout, A.-L. (2015). Les transformations du milieu scolaire spécialisé: le cas des écoles d'aveugles en France et au Japon. La nouvelle revue de l'adaptation et de la scolarisation, 69, 157-167.

OMS. (2012). Global data on visual impairments 2010. [En ligne].

Onisep. (2014). Annuaire jeunes handicapés visuels. Paris: Onisep.

Puustinen, M., Arneton, M., Lewi-Dumont, N. et Gaborit, M. (accepté). Help seeking in students with a visual impairment: Students' and teachers' perspective. Communication orale, $16^{e}$ conférence bisannuelle d'European Association for Research on Learning and Instruction (EARLI), Limassol (Chypre), 25-29 août 2015.

Puustinen, M., Lewi-Dumont, N., Arneton, M. et Cuer-Buard, K. (2013). Help seeking in students with special educational needs: The case of visual impairment. Communication orale, $15^{\mathrm{e}}$ conférence bisannuelle d'European Association for Research on Learning and Instruction (EARLI), Munich (Allemagne), $27-31$ août. Rick, O. et Ebersold, S. (2011). Lycéen, et après? Les parcours vers l'enseignement supérieur et l'emploi. Suresne: INS HEA.

Sémelin, J. (2007). J'arrive où je suis étranger. Paris: Seuil.

Toubert, D. et Bartolucci, A. (2015, à paraître). La construction de l'espace chez l'enfant déficient visuel. In N. Lewi-Dumont (dir.). Enseigner à des élèves malvoyants, enseigner à des élèves aveugles. Lille: Canopé. 